\title{
Veterinary World reviewer acknowledgment 2017
}

\author{
A. V. Sherasiya and Nazir
}

Veterinary World, Star, Gulshan Park, NH-8A, Chandrapur Road, Wankaner - 363621, Dist. Morbi, Gujarat, India. Corresponding author: A. V. Sherasiya, e-mail: editorveterinaryworld@gmail.com,

Nazir: veterinaryworldpublisher@gmail.com Received: 01-01-2018, Published online: 12-01-2018

doi: 10.14202/vetworld.2018.10-13 How to cite this article: Sherasiya AV, Nazir (2018) Veterinary World reviewer acknowledgment 2017, Veterinary World, 11(1): 10-13.

\section{Contributing reviewers}

Veterinary World editorial team would sincerely like to thank all of our reviewers who contributed to peer review for the journal in 2017.

\author{
A. Aygun \\ Turkey
}

A. K. M. Anisur Rahman

Bangladesh

\section{Abdelaziz ED-DRA \\ Morocco}

\section{Abid Hussain Shahzad \\ Pakistan}

\section{Ahmed Ali Mahmoud Saleh Japan}

\author{
Ahmed S Abdoon \\ Egypt
}

\section{Ailian Geng \\ China}

\section{Alfredo Medrano \\ Mexico}

Amira Taman

Egypt

Amlan K. Patra

India

Ammar Ahmad Khan

Pakistan

Ana Paula Loureiro

Brazil

Anand S. P.

India

Andres Carvajal
Anna Faltýnková

Czech Republic

Annamaria Passantino

Italy

Antoni Prenafeta

Spain

Antonio Varcasia

Italy

\section{Anut Chantiratikul}

Thailand

Arathy D. S. Nair

USA

Arbouche Rafik

Algeria

Archana Jain

India

Archana Verma

India

Arda Sozcu

Turkey

Arda Yildirim

Turkey

Aripudur Sellappan Rajendiran India

Arun Kumar De
India

Arya Sobhakumari

USA

\section{Ashish Chopra \\ India}

Ashwani Kumar

India

Aspinas Chapwanya

Kenya

B. Madhusudhana Rao

India

\section{Bala Manickam}

USA

Balamurugan Vinayagamurthy India

Bambang Pontjo Priosoeryanto Indonesia

Bartosz Kierończyk

Poland

Ben Adler

Australia

Bersissa Kumsa Eseta

Ethiopia

Brad M Isaacson

USA

Bryony A. Jones

UK

Buket Er Demirhan

Turkey

C. E. Atkins

USA

Copyright: Sherasiya and Nazir. Open Access. This article is distributed under the terms of the Creative Commons Attribution 4.0 International License (http://creativecommons.org/licenses/by/4.0/), which permits unrestricted use, distribution, and reproduction in any medium, provided you give appropriate credit to the original author(s) and the source, provide a link to the Creative Commons license, and indicate if changes were made. The Creative Commons Public Domain Dedication waiver (http://creativecommons.org/publicdomain/zero/1.0/) applies to the data made available in this article, unless otherwise stated. 


\begin{tabular}{|c|c|c|}
\hline $\begin{array}{l}\text { Chaleow Salakij } \\
\text { Thailand }\end{array}$ & $\begin{array}{l}\text { Eryk Abdul Rantam } \\
\text { Indonesia }\end{array}$ & $\begin{array}{l}\text { Hazem Shaheen } \\
\text { Egypt }\end{array}$ \\
\hline $\begin{array}{l}\text { Chanchal Guha } \\
\text { India }\end{array}$ & $\begin{array}{l}\text { Fabio De Rensis } \\
\text { Italy }\end{array}$ & $\begin{array}{l}\text { Hua-Ji Qiu } \\
\text { China }\end{array}$ \\
\hline $\begin{array}{l}\text { Chandramani Pathak } \\
\text { India }\end{array}$ & $\begin{array}{l}\text { Felipp Silveira Ferreira } \\
\text { Brazil }\end{array}$ & $\begin{array}{l}\text { Hudaa Neetoo } \\
\text { Mauritius }\end{array}$ \\
\hline $\begin{array}{l}\text { Christian Tiambo Keambou } \\
\text { Kenya }\end{array}$ & $\begin{array}{l}\text { Filippo Giarratana } \\
\text { Italy }\end{array}$ & $\begin{array}{l}\text { Hudson A. Pinto } \\
\text { Brazil }\end{array}$ \\
\hline $\begin{array}{l}\text { Christopher Didigwu Nwani } \\
\text { Nigeria }\end{array}$ & $\begin{array}{l}\text { Florence D Tarfa } \\
\text { Nigeria }\end{array}$ & $\begin{array}{l}\text { Hugo Leonardo da Cunha } \\
\text { Amaral } \\
\text { Brazil }\end{array}$ \\
\hline $\begin{array}{l}\text { Chyer Kim } \\
\text { USA }\end{array}$ & $\begin{array}{l}\text { Fufa Ido Gimba } \\
\text { Malaysia }\end{array}$ & $\begin{array}{l}\text { Huiling Sun } \\
\text { China }\end{array}$ \\
\hline $\begin{array}{l}\text { Claudia Bezerra da Silva } \\
\text { Brazil }\end{array}$ & $\begin{array}{l}\text { Gamal Wareth } \\
\text { Germany }\end{array}$ & $\begin{array}{l}\text { Ionel D. Bondoc } \\
\text { Romania }\end{array}$ \\
\hline $\begin{array}{l}\text { Coen M. Adema } \\
\text { USA }\end{array}$ & $\begin{array}{l}\text { Genevieve Andre-Fontaine } \\
\text { France }\end{array}$ & $\begin{array}{l}\text { Irena Musik } \\
\text { Poland }\end{array}$ \\
\hline $\begin{array}{l}\text { Cromwell Purchase } \\
\text { Qatar }\end{array}$ & $\begin{array}{l}\text { Gitao Chege George } \\
\text { Kenya }\end{array}$ & $\begin{array}{l}\text { Ismail Hakkı Tekiner } \\
\text { Turkey }\end{array}$ \\
\hline $\begin{array}{l}\text { David Kennedy } \\
\text { USA }\end{array}$ & $\begin{array}{l}\text { Giuseppe Piccone } \\
\text { Italy }\end{array}$ & $\begin{array}{l}\text { James Satvil Dalis } \\
\text { Nigeria }\end{array}$ \\
\hline $\begin{array}{l}\text { Deepti Chachra } \\
\text { India }\end{array}$ & $\begin{array}{l}\text { Gnanavel Venkatesan } \\
\text { India }\end{array}$ & $\begin{array}{l}\text { Jason Devoy Keegan } \\
\text { Ireland }\end{array}$ \\
\hline $\begin{array}{l}\text { Denisa Soledad Pérez Gaudio } \\
\text { Argentina }\end{array}$ & $\begin{array}{l}\text { Guilherme Dias de Melo } \\
\text { France }\end{array}$ & $\begin{array}{l}\text { Jayraw A. K. } \\
\text { India }\end{array}$ \\
\hline $\begin{array}{l}\text { Dinesh M. D. } \\
\text { India }\end{array}$ & $\begin{array}{l}\text { Guillermo Roberto Risatti } \\
\text { USA }\end{array}$ & $\begin{array}{l}\text { Jebreil shams shamseddin } \\
\text { Iran }\end{array}$ \\
\hline $\begin{array}{l}\text { Dragica Salamon } \\
\text { Croatia }\end{array}$ & $\begin{array}{l}\text { Gul Ahmad } \\
\text { USA }\end{array}$ & $\begin{array}{l}\text { Jerome Andonissamy } \\
\text { India }\end{array}$ \\
\hline $\begin{array}{l}\text { Duane L. Garner Garner } \\
\text { USA }\end{array}$ & $\begin{array}{l}\text { Guoxiang Chao } \\
\text { China }\end{array}$ & $\begin{array}{l}\text { Jitendra Goswami } \\
\text { India }\end{array}$ \\
\hline $\begin{array}{l}\text { E. Carretón } \\
\text { Spain }\end{array}$ & $\begin{array}{l}\text { Guo-zhong Zhang } \\
\text { China }\end{array}$ & $\begin{array}{l}\text { João Simões } \\
\text { Portugal }\end{array}$ \\
\hline $\begin{array}{l}\text { Ehsan Ahmadpour } \\
\text { Iran }\end{array}$ & $\begin{array}{l}\text { Gwendolyn L Carroll } \\
\text { USA }\end{array}$ & $\begin{array}{l}\text { Jose Edmundo Apraez Guerrero } \\
\text { Colombia }\end{array}$ \\
\hline $\begin{array}{l}\text { Elif Çelik } \\
\text { Turkey }\end{array}$ & $\begin{array}{l}\text { Hanna Markiewicz } \\
\text { Poland }\end{array}$ & $\begin{array}{l}\text { Joseph Bosilevac } \\
\text { USA }\end{array}$ \\
\hline $\begin{array}{l}\text { Elisabetta Manuali } \\
\text { Italy }\end{array}$ & $\begin{array}{l}\text { Hari Mohan Saxena } \\
\text { India }\end{array}$ & $\begin{array}{l}\text { Joshua Oluoch Amimo } \\
\text { Kenya }\end{array}$ \\
\hline $\begin{array}{l}\text { Emilia Bagnicka } \\
\text { Poland }\end{array}$ & $\begin{array}{l}\text { Hasan Meydan } \\
\text { Turkey }\end{array}$ & $\begin{array}{l}\text { K. G. Meade Meade } \\
\text { Ireland }\end{array}$ \\
\hline $\begin{array}{l}\text { Erma Safitri } \\
\text { Indonesia }\end{array}$ & $\begin{array}{l}\text { Hassan Borji } \\
\text { Iran }\end{array}$ & $\begin{array}{l}\text { Kai Huang } \\
\text { USA }\end{array}$ \\
\hline
\end{tabular}




\begin{tabular}{|c|c|c|}
\hline $\begin{array}{l}\text { Kannan Thandavan Arthanari } \\
\text { India }\end{array}$ & $\begin{array}{l}\text { Md Fakruddin } \\
\text { Bangladesh }\end{array}$ & $\begin{array}{l}\text { Nicholas Johnson } \\
\text { UK }\end{array}$ \\
\hline $\begin{array}{l}\text { Karim El-Sabrout } \\
\text { Egypt }\end{array}$ & $\begin{array}{l}\text { Mehmet Fatih Aydin } \\
\text { Turkey }\end{array}$ & $\begin{array}{l}\text { Nirupama Agrawal } \\
\text { India }\end{array}$ \\
\hline $\begin{array}{l}\text { Kirstine Klitgaard Schou } \\
\text { Denmark }\end{array}$ & $\begin{array}{l}\text { Mian Muhammad Awais } \\
\text { Pakistan }\end{array}$ & $\begin{array}{l}\text { Nitesh Kumar } \\
\text { India }\end{array}$ \\
\hline $\begin{array}{l}\text { Kolluri Gautham } \\
\text { India }\end{array}$ & $\begin{array}{l}\text { Michael M. Schutz } \\
\text { USA }\end{array}$ & $\begin{array}{l}\text { Noha Salem } \\
\text { Egypt }\end{array}$ \\
\hline $\begin{array}{l}\text { Kosta Y. Mumcuoglu } \\
\text { Israel }\end{array}$ & $\begin{array}{l}\text { Miguel Quaresma } \\
\text { Portugal }\end{array}$ & $\begin{array}{l}\text { Nuh Kiliç } \\
\text { Turkey }\end{array}$ \\
\hline $\begin{array}{l}\text { Koushlesh Ranjan } \\
\text { India }\end{array}$ & $\begin{array}{l}\text { Minakshi Prasad } \\
\text { India }\end{array}$ & $\begin{array}{l}\text { Oludayo O Fashina } \\
\text { South Africa }\end{array}$ \\
\hline $\begin{array}{l}\text { Laurent Falquet } \\
\text { Switzerland }\end{array}$ & $\begin{array}{l}\text { Mobini Behzad } \\
\text { Iran }\end{array}$ & $\begin{array}{l}\text { Osama Ibrahim Azawi } \\
\text { Iraq }\end{array}$ \\
\hline $\begin{array}{l}\text { Laxmi Narayan Sarangi } \\
\text { India }\end{array}$ & $\begin{array}{l}\text { Mohamed Haroun - Haroun } \\
\text { Qatar }\end{array}$ & $\begin{array}{l}\text { Panagiotis E Simitzis } \\
\text { Greece }\end{array}$ \\
\hline $\begin{array}{l}\text { Lenin Lenin Aguirre Riofrio } \\
\text { Ecuador }\end{array}$ & $\begin{array}{l}\text { Mohammad Mijanur Rahman } \\
\text { Malaysia }\end{array}$ & $\begin{array}{l}\text { Panch Kishor Bharti } \\
\text { India }\end{array}$ \\
\hline $\begin{array}{l}\text { Liben Chen } \\
\text { USA }\end{array}$ & $\begin{array}{l}\text { Mohammad Taghi Rahimi } \\
\text { Iran }\end{array}$ & $\begin{array}{l}\text { Parag Nigam } \\
\text { India }\end{array}$ \\
\hline $\begin{array}{l}\text { Liliana Aguilar-Marcelino } \\
\text { Mexico }\end{array}$ & $\begin{array}{l}\text { Mohammed Hamdy Farouk } \\
\text { China }\end{array}$ & $\begin{array}{l}\text { Paramanandham } \\
\text { Krishnamoorthy } \\
\text { India }\end{array}$ \\
\hline $\begin{array}{l}\text { Linous Munsimbwe } \\
\text { Zambia }\end{array}$ & $\begin{array}{l}\text { Mohan Maddur } \\
\text { USA }\end{array}$ & $\begin{array}{l}\text { Pinaki Prasad Sengupta } \\
\text { India }\end{array}$ \\
\hline $\begin{array}{l}\text { Livia Ribeiro Mendonca } \\
\text { Brazil } \\
\text { Luis Souza Lima de Souza Reis }\end{array}$ & $\begin{array}{l}\text { Mohie Abdallah Mahmoud } \\
\text { Haridy } \\
\text { Japan }\end{array}$ & $\begin{array}{l}\text { R. Mirmahmoudi } \\
\text { Iran }\end{array}$ \\
\hline $\begin{array}{l}\text { Brazil } \\
\text { Mahmoud Mohey Elhaig }\end{array}$ & $\begin{array}{l}\text { Moustafa kardjadj } \\
\text { Algeria }\end{array}$ & $\begin{array}{l}\text { Rachel BINET } \\
\text { USA }\end{array}$ \\
\hline $\begin{array}{l}\text { Egypt } \\
\text { Manjunatha Bodhaganahalli }\end{array}$ & $\begin{array}{l}\text { Moutaz Zarkawi } \\
\text { Syria }\end{array}$ & $\begin{array}{l}\text { Rafael Cardoso Carvalho } \\
\text { Brazil }\end{array}$ \\
\hline $\begin{array}{l}\text { Oman } \\
\text { Manjunatha Narayanappa }\end{array}$ & $\begin{array}{l}\text { N. De Briyne } \\
\text { Belgium }\end{array}$ & $\begin{array}{l}\text { Rafiqul Islam } \\
\text { India }\end{array}$ \\
\hline $\begin{array}{l}\text { Belaganahalli } \\
\text { USA }\end{array}$ & $\begin{array}{l}\text { N. Punniamurthy, Natesan } \\
\text { India }\end{array}$ & $\begin{array}{l}\text { Rahul Nandre } \\
\text { USA }\end{array}$ \\
\hline $\begin{array}{l}\text { Maria Emilia Eirin } \\
\text { Argentina }\end{array}$ & $\begin{array}{l}\text { Nadeem Shabir } \\
\text { India }\end{array}$ & $\begin{array}{l}\text { Rajani Ravindranath Thanissery } \\
\text { USA }\end{array}$ \\
\hline $\begin{array}{l}\text { Mário Manuel Dinis Ginja } \\
\text { Portugal }\end{array}$ & $\begin{array}{l}\text { Naim Deniz Ayaz } \\
\text { Turkey }\end{array}$ & $\begin{array}{l}\text { Raquel Salazar Lugo } \\
\text { Venezuela }\end{array}$ \\
\hline $\begin{array}{l}\text { Martin M Nyaga } \\
\text { South Africa }\end{array}$ & $\begin{array}{l}\text { Nazarudin M.F. } \\
\text { Malaysia }\end{array}$ & $\begin{array}{l}\text { Ravi Kant Upadhyay } \\
\text { India }\end{array}$ \\
\hline $\begin{array}{l}\text { Masayuki Shimada } \\
\text { Japan }\end{array}$ & $\begin{array}{l}\text { Nazli Ercan } \\
\text { Turkey }\end{array}$ & $\begin{array}{l}\text { Rekha Sharma } \\
\text { India }\end{array}$ \\
\hline
\end{tabular}




\begin{tabular}{|c|c|c|}
\hline $\begin{array}{l}\text { Reza Masoumi } \\
\text { Iran }\end{array}$ & $\begin{array}{l}\text { Sreenu Makkena } \\
\text { India }\end{array}$ & $\begin{array}{l}\text { Walied Abdo } \\
\text { Egypt }\end{array}$ \\
\hline $\begin{array}{l}\text { Richa Sood } \\
\text { India }\end{array}$ & $\begin{array}{l}\text { Stefania Perrucci } \\
\text { Italy }\end{array}$ & $\begin{array}{l}\text { Widya Paramita Lokapirnasari } \\
\text { Indonesia }\end{array}$ \\
\hline $\begin{array}{l}\text { Rodrigo Alberto Jerez } \\
\text { Ebensperger } \\
\text { Spain }\end{array}$ & $\begin{array}{l}\text { Süleyman Cilek } \\
\text { Turkey }\end{array}$ & $\begin{array}{l}\text { William Alberto Cañón-Franco } \\
\text { Colombia }\end{array}$ \\
\hline $\begin{array}{l}\text { Rolf karl Schuster } \\
\text { United Arab Emirates }\end{array}$ & $\begin{array}{l}\text { Sunil More } \\
\text { USA }\end{array}$ & $\begin{array}{l}\text { Wojciech Barański } \\
\text { Poland }\end{array}$ \\
\hline $\begin{array}{l}\text { Rui Wu } \\
\text { China }\end{array}$ & $\begin{array}{l}\text { Sven Kaese } \\
\text { Germany }\end{array}$ & $\begin{array}{l}\text { Ya-Ching Lin } \\
\text { Taiwan }\end{array}$ \\
\hline $\begin{array}{l}\text { S. R. Rüegg } \\
\text { Czech Republic }\end{array}$ & $\begin{array}{l}\text { T. Madani Toufik } \\
\text { Algeria }\end{array}$ & $\begin{array}{l}\text { Ya-Ling Huang } \\
\text { Taiwan }\end{array}$ \\
\hline $\begin{array}{l}\text { Sameer D Pant } \\
\text { Australia }\end{array}$ & $\begin{array}{l}\text { Tanko Polycarp Nwunuji } \\
\text { Nigeria }\end{array}$ & $\begin{array}{l}\text { Yasser Mahmmod } \\
\text { Denmark }\end{array}$ \\
\hline Sandeep Ghatak & $\begin{array}{l}\text { Thomas J Nolan } \\
\text { USA }\end{array}$ & $\begin{array}{l}\text { Youssef A. Attia } \\
\text { Saudi Arabia }\end{array}$ \\
\hline Satoshi Sekiguchi & $\begin{array}{l}\text { Tomas Erban } \\
\text { Czech Republic }\end{array}$ & $\begin{array}{l}\text { Yukita Sato } \\
\text { Japan }\end{array}$ \\
\hline Seyyed Khalil Hosseinihashemi & $\begin{array}{l}\text { Ummey Shameem } \\
\text { India }\end{array}$ & $\begin{array}{l}\text { Yves Millemann } \\
\text { France }\end{array}$ \\
\hline Shailbala Singh & $\begin{array}{l}\text { V. C. Rayulu } \\
\text { India }\end{array}$ & $\begin{array}{l}\text { Zahid Naseer } \\
\text { Pakistan }\end{array}$ \\
\hline $\begin{array}{l}\text { Shoja Jafari } \\
\text { Iran }\end{array}$ & $\begin{array}{l}\text { Valeria De Cesaris } \\
\text { Italy }\end{array}$ & $\begin{array}{l}\text { Zeki Aras } \\
\text { Turkey }\end{array}$ \\
\hline $\begin{array}{l}\text { Silvana Scarcella } \\
\text { Argentina }\end{array}$ & $\begin{array}{l}\text { Varij Nayan } \\
\text { India }\end{array}$ & $\begin{array}{l}\text { Zuhair Ismail } \\
\text { Jordan }\end{array}$ \\
\hline $\begin{array}{l}\text { Sinan Ince } \\
\text { Turkey }\end{array}$ & $\begin{array}{l}\text { Vera Mores Rall } \\
\text { Brazil }\end{array}$ & $\begin{array}{l}\text { Zygmunt Kazimierz Giżejewski } \\
\text { Poland }\end{array}$ \\
\hline $\begin{array}{l}\text { Sorin Daniel Dan } \\
\text { Romania }\end{array}$ & $\begin{array}{l}\text { Vihang Vithalrao Patil } \\
\text { India } \\
\text { Vishal S Suthar }\end{array}$ & \\
\hline $\begin{array}{l}\text { Sreekumar Chirukandoth } \\
\text { India }\end{array}$ & India & \\
\hline
\end{tabular}

